\title{
Combination of measurement uncertainty contributions via the generalized Dombi operator
}

\author{
Alessandro Ferrero ${ }^{1}$ Marco Prioli $^{1}$ Simona Salicone $^{1}$ Wei Jiang ${ }^{2}$ \\ ${ }^{1}$ Department of Electronics, Information and Bioengineering - Politecnico di Milano \\ Piazza Leonardo da Vinci, 32 - 20133 Milano - Italy \\ ${ }^{2}$ Department of Instrument Science and Technology - College of Mechatronics and Automation \\ National University of Defense Technology \\ Changsha city - Hunan Province - P.R. China - 410073
}

\begin{abstract}
Possibility is considered as a promising mathematical tool to represent that particular case of incomplete information given by a measurement result. A measurement result can be affected by systematic and random effects. The combination of random contributions in the possibility theory returns only approximated results, and it is important to identify an optimal combination method to obtain acceptable uncertainty evaluations. This paper considers the generalized Dombi operator as a promising tool to obtain satisfying results.
\end{abstract}

Keywords: Measurement Uncertainty. Random contributions. Probability. Possibility. $t$-norms. Generalized Dombi Operator.

\section{Introduction}

It is now universally recognized $[1,2]$ that the result of a measurement provides only a finite amount of information about the measurand. Therefore, a measurement result is useless unless some additional relevant information [3] can be associated to the result itself, aimed at quantifying how complete this information is. Today, measurement uncertainty represents this additional relevant information.

The present practice of measurement, encompassed in two official documents issued by the $\mathrm{Bu}-$ reau International des Poids et Mesures (BIPM), the International Vocabulary of Metrology (VIM) [3] and the Guide to the expression of Uncertainty in Measurement (GUM) [1], prescribes that all significant systematic effects are recognized and compensated for, so that only random effects are assumed to affect the measurement process and contribute to measurement uncertainty. Under this assumption, probability is the most effective mathematical tool to represent the incomplete information provided by a measurement result, that is therefore represented by a probability distribution, characterized by its first two moments, the mean (measured value) and its standard deviation (standard uncertainty) [1].

However, in many industrial processes, the identification and compensation of the systematic effects may become a too expensive task, so that it is important to take into account also the systematic contributions to uncertainty and combine them with all other contributions, to evaluate the overall measurement uncertainty and assess whether these effects can be tolerated or have to be compensated.

For this reason, the possibility theory has been recently investigated as a more general tool than probability to express measurement results together with the related measurement uncertainty $[2,4,5]$, and Random-Fuzzy Variables (RFVs) have been defined $[6,7]$ as a promising tool for representing and combining measurement results and related uncertainty.

While possibility allows one to represent and combine systematic contributions in a strict way, when used to combine random contributions it can only provide approximate solutions $[2,8]$ to the strict ones obtained in the probability domain. To keep the advantages of a unique approach to measurement uncertainty, capable of representing and processing all contributions to uncertainty, it is then important to define an optimal way to combine random contributions in the possibility domain.

This paper considers the generalized Dombi operator [9] and shows that it can provide satisfactory approximations in many cases of interest in the measurement field, much better than the ones previously obtained by the same authors employing single-parameter $t$-norm families [8].

\section{Uncertainty representation}

Within the RFV approach [2,6,7], the RFV itself is capable of representing the result of a measurement, i.e. the best measurand estimate together with the associated estimate of measurement uncertainty. An example of RFV is shown in the last plot of Fig. 1. This variable is composed by two dif- 

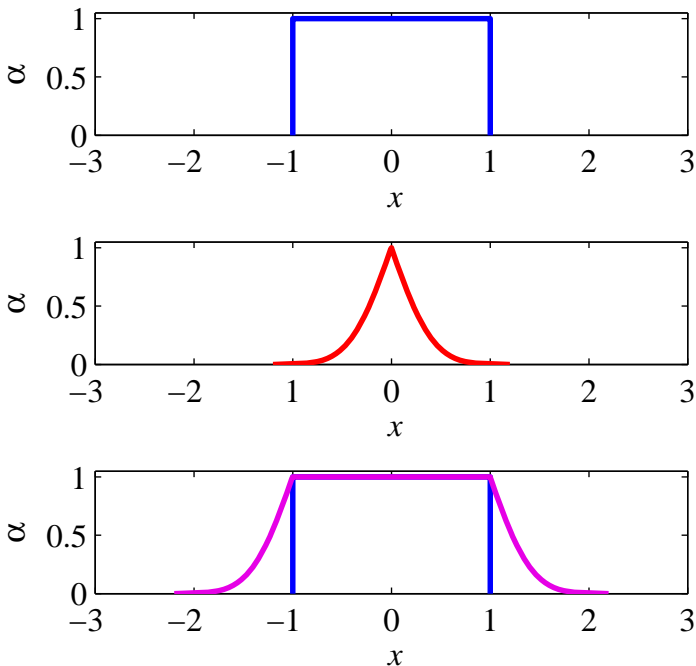

Figure 1: Internal PD $r_{X}^{\text {int }}$ (blue line), random PD $r_{X}^{\mathrm{ran}}$ (red line) and resulting RFV of $X$ (blue and magenta lines).

ferent possibility distributions (PDs): an "internal PD" $r$ int (blue line in Fig. 1), which represents the non-random contributions to uncertainty (e.g. the contribution of uncompensated systematic effects), and an "external PD" $r^{\text {ext }}$ (magenta line in Fig. 1), which represents all contributions to uncertainty. The external PD is obtained by combining $r^{\text {int }}$ with a "random PD" $r^{\text {ran }}$ (red line in Fig. 1), which represents only the random contributions to uncertainty (e.g. the contribution of random noise).

According to [10], the available metrological information about a measured quantity $X$ leads to the construction of $r_{X}^{\text {int }}$ and $r_{X}^{\text {ran }}$. In particular, the PD $r_{X}^{\text {int }}$ is generally obtained directly as a PD starting from the available evidence [11]. On the other hand, since the available evidence about random contributions is commonly represented by means of a probability density function $(\mathrm{PDF}) p_{X}, r_{X}^{\mathrm{ran}}$ is often obtained by means of a probability-possibility transformation $[12,13]$ applied to $p_{X}$. Starting from $r_{X}^{\text {int }}$ and $r_{X}^{\mathrm{ran}}$, the external PD $r_{X}^{\mathrm{ext}}$ can be derived [14], as:

$$
r_{X}^{\mathrm{ext}}(x)=\sup _{x^{\prime}} T_{m}\left[r_{X}^{\mathrm{ran}}\left(x-x^{\prime}+x^{*}\right), r_{X}^{\mathrm{int}}\left(x^{\prime}\right)\right]
$$

where $x^{*}$ is the mode of $r_{X}^{\mathrm{ran}}$ and $T_{m}$ is the min $t$-norm [15]. Equation (1) can be interpreted as follows: the external possibility $r_{X}^{\text {ext }}$ is, for each $x$ value, the sup of $r_{X}^{\text {ran }}$ centered on a new mode value $x^{\prime}$, limited by the possibility that $x^{\prime}$ represents a new mode value $\left(r_{X}^{\text {int }}\left(x^{\prime}\right)\right)$.

According to $[2,7]$, the cuts at level $\alpha$ ( $\alpha$-cuts) of $r_{X}^{\text {ext }}$ generalize the probabilistic concept of confidence intervals. Their confidence level can be simply obtained as $\gamma=1-\alpha$.

\section{Uncertainty combination}

Within the RFV approach $[2,7]$, uncertainty is propagated through the combination of PDs. In general, PDs can be combined by means of the Zadeh's extension principle (ZEP) [16]:

$$
r_{Z}(z)=\sup _{z=f(x, y)} r_{X, Y}(x, y)
$$

From the measurement point of view, this principle can be interpreted as follows: starting from the joint PD $r_{X, Y}$ associated with the measured variables $X$ and $Y$, the ZEP provides the PD $r_{Z}$ associated with measurand $Z=f(X, Y)$. Therefore, $f$ represents a generic measurement function.

To obtain $r_{Z}$, the joint PD $r_{X, Y}$ has to be first evaluated starting from the available metrological information $[8,11]$. In most cases, the available metrological information concerns the possible values of the measured variables and their relation and leads, as shown in [17], to the construction of the marginal PD $r_{X}$ and the conditional PD $r_{Y \mid X}$, already defined in [18]. Once the distributions $r_{X}$ and $r_{Y \mid X}$ are obtained, the joint PD can be found as:

$$
r_{X, Y}(x, y)=T\left[r_{X}(x), r_{Y \mid X}(y \mid x)\right]
$$

$T$ being a $t$-norm $[15,19]$. Of course, if evidence about the independence of the measured variables $X$ and $Y$ is available, (3) simplifies to:

$$
r_{X, Y}(x, y)=T\left[r_{X}(x), r_{Y}(y)\right]
$$

The choice of the specific $t$-norm to be applied in (3) and (4) represents the most critical point in the definition of the uncertainty combination method within the RFV approach. In fact, two different $t$ norms can lead to two significantly different joint PDs, and, therefore, two different PDs associated with measurand $Z$ are obtained by means of (2). For this reason, a criterion has been identified in $[8,11]$ to find the optimal $t$-norm for the combination of measurement uncertainty.

The referenced papers state that the choice of a specific $t$-norm depends on the nature of the uncertainty contributions represented by the PDs, i.e. that two different $t$-norms have to be considered for the combination of internal PDs and random PDs. The fact that internal PDs represent the uncertainty contributions due to all kinds of nonrandom effects led, in [11], to the conclusion that a minimum specificity principle (or maximum entropy principle) should be followed for the determination of the specific $t$-norm for their aggregation. Of course, the maximum entropy in their aggregation is obtained by selecting the least specific $t$-norm, i.e. the min $t$-norm $T_{m}[15,19]$.

On the other hand, the fact that random PDs represent the uncertainty contributions due to the sole random effects led, in [8], to the conclusion that a maximum specificity principle (or minimum entropy 
principle) should be followed for the determination of the specific $t$-norm for their aggregation. The minimum entropy in their aggregation can be obtained by selecting a $t$-norm among the Frank parametric family $[19,20]$ :

$$
\begin{aligned}
& T_{\gamma}^{F}\left[r_{1}, r_{2}\right]= \\
& \quad= \begin{cases}T_{m}\left[r_{1}, r_{2}\right] & \text { if } \gamma=0 \\
T_{p}\left[r_{1}, r_{2}\right] & \text { if } \gamma=1 \\
T_{L}\left[r_{1}, r_{2}\right] & \text { if } \gamma=\infty \\
\log _{\gamma}\left(1+\frac{\left(\gamma^{r_{1}}-1\right) \cdot\left(\gamma^{r_{2}}-1\right)}{\gamma-1}\right) & \text { else }\end{cases}
\end{aligned}
$$

where $T_{p}$ is the product $t$-norm, $T_{L}$ is the Lukasiewicz's $t$-norm $[15,19]$ and $\gamma$ is the parameter value.

While the criterion identified in [11] for the internal PDs leads to a single $t$-norm, the criterion identified in [8] for the random PDs leads to a $t$-norm parametric family. This means that an optimum parameter value has to be identified for the combination of random PDs, i.e. for the combination of random contributions to uncertainty.

A numerical method has been devised in [8] to identify the optimum parameter value, comparing the combination of the random contributions that is obtained in the possibility domain for a given parameter $\gamma$ with the combination of the same contributions in their natural domain: the probability domain. According to this method, when random contributions are assumed to be normally distributed, quite similar result are obtained in the two domains for $\gamma=0.05$.

The application of this $t$-norm to particular cases $[14,21]$ yielded good results, but also showed that, even considering the optimum parameter value $\gamma=$ 0.05, small differences appear between the final uncertainty estimate obtained in the two domains. In other words, the approach devised for the combination of random contributions in the possibility domain is only a numerical approximation of the (correct) combination of the same contributions in the probability domain. This numerical approximation shows it weakness when several random contributions have to be combined [21]. In fact, in this case, the final uncertainty value obtained in the possibility domain may result in a underestimate of the correct uncertainty value provided by the probability domain.

Therefore, when several random contributions have to be combined, the simplicity of a single parameter $t$-norm family has to be abandoned, and more complex and flexible $t$-norms have to be investigated. A good candidate for the combination of multiple random PDs appears to be the general- ized Dombi operator (GDO) [9]:

$$
\begin{aligned}
& T_{\gamma_{1}, \gamma_{2}}^{\mathrm{GDO}}[\mathbf{r}]= \\
& =\frac{1}{1+\left(\frac{1}{\gamma_{1}}\left(\prod_{i=1}^{N}\left(1+\gamma_{1}\left(\frac{1-r_{i}}{r_{i}}\right)^{\gamma_{2}}\right)-1\right)\right)^{1 / \gamma_{2}}}
\end{aligned}
$$

where $\mathbf{r}$ is a vector of $N$ possibility distributions $r_{i}$. As shown by $(6), T_{\gamma_{1}, \gamma_{2}}^{\mathrm{GDO}}$ is a two-parameter $t$ norm family, i.e. it shows an additional freedom degree with respect to the Frank family. Therefore, it is worth checking here if this operator can lead to a more accurate combination of random contributions to uncertainty. In the next Sections, the procedure for the identification of the best $t$-norm for the combination of random PDs and the optimal $t$-norm parameters will be briefly recalled.

\subsection{Identification of the optimal $t$-norm}

The $t$-norm selection procedure is based on the the availability of a specifically designed 2-D probability-possibility transformation. This transformation has been introduced in [13] in order to preserve the maximum possible amount of metrological information in the transformation from a joint PDF to a joint PD. In particular, [13] has proved that the maximum amount of information about the marginal distributions of the joint distributions and their possible correlation is preserved in the transformation. Moreover, [13] has proved that also the information about a variable $Z$ derived from $X$ and $Y$ is preserved.

To clarify this point, let us consider a bivariate standard normal $\operatorname{PDF} p_{X, Y}=\mathcal{N}_{2}(\mathbf{0}, \mathbf{I})$ and a variable $Z$ derived from $X$ and $Y$, as $Z=X+Y$. According to [13], if a joint $\mathrm{PD} \hat{r}_{X, Y}$ is derived from $p_{X, Y}$ by means of the 2 -D probability-possibility transformation defined in [13], $p_{Z}$ obtained from the joint PDF $p_{X, Y}$ and $r_{Z}$ obtained from the joint PD $\hat{r}_{X, Y}$ through (2) provide the same information about $Z$.

According to the above discussion, the joint PD $\hat{r}_{X, Y}$ provided by this transformation can serve as a reference joint $\mathrm{PD}$ in the definition of the best $t$ norm for the combination of random contributions in the possibility domain. Let us assume that the marginal distributions $p_{X}$ and $p_{Y}$ of $p_{X, Y}$ are transformed into their equivalent possibility distributions $r_{X}$ and $r_{Y}$ by means of the 1-D transformation discussed in $[12,13]$. Moreover, let us assume that $r_{X}$ and $r_{Y}$ are combined into a joint PD $\tilde{r}_{X, Y}$ by means of a $t$-norm $\tilde{T}$, according to (4). Starting from these assumptions, from a strict theoretical point of view, the optimal $t$-norm $\tilde{T}$ for the combination of random contributions is the $t$-norm yielding:

$$
\tilde{r}_{X, Y}=\hat{r}_{X, Y}
$$

In fact, only in this unique case, the proof given in [13] ensures that equivalent distributions of $Z$ 


\begin{tabular}{|c|c|c|}
\hline$t$-norm & Param. values & $e$ \\
\hline$T_{\gamma}^{F}$ & $\gamma=0.05$ & $3.4 \%$ \\
$T_{\gamma_{1}, \gamma_{2}}^{\mathrm{GDO}}$ & $\gamma_{1}=0.55$ & \\
& $\gamma_{2}=1.47$ & $0.9 \%$ \\
\hline
\end{tabular}

Table 1: Errors $e$ introduced by $T_{\gamma}^{F}$ and $T_{\gamma_{1}, \gamma_{2}}^{\mathrm{GDO}}$. Standard Normal distributions are assumed for $p_{X}$ and $p_{Y}$.

are obtained in the probability and the possibility domains, i.e. that, starting from equivalent assumptions about the random contributions, the same uncertainty estimate is obtained in the two domains.

\subsection{Identification of the optimal $t$-norms parameters}

Unfortunately, a closed form expression of an operator $\tilde{T}$ strictly satisfying (7) has not yet been found. Therefore, the most common parametric families of $t$-norms have been considered in [8] as the optimal operator $\tilde{T}$. In general, the considered $t$-norm families do not satisfy (7), i.e. the resulting joint $\mathrm{PD}$ can be different than the reference joint $\mathrm{PD}$. This means that the considered $t$-norm families introduce an error in the combination of random contributions.

Of course, the error introduced by the $t$-norm parametric families is a function of their parameters. Therefore, the parameter values for which the minimum errors are obtained have to be identified. A numerical procedure can be followed to obtain the optimal $t$-norms parameters. This procedure is based on the following definition of normalized squared error:

$$
e=\sqrt{\frac{\iint\left(\hat{r}_{X, Y}(x, y)-\tilde{r}_{X, Y}(x, y)\right)^{2} d x d y}{\iint \hat{r}_{X, Y}(x, y)^{2} d x d y}}
$$

When this procedure is applied to the Frank $t$ norm family, the optimal parameter value and the minimum error $e$ reported in Table 1 are obtained. The optimization procedure can be applied also to the GDO, though in this case two parameters must be optimized. The optimal parameters and the minimum error for the GDO are also reported in Table 1. Comparing the two errors, it can be immediately seen that the GDO represents an important improvement in the combination of the random contributions in the possibility domain. In fact, its capability of approximating the reference joint $\mathrm{PD}$ is significantly better than the Frank $t$-norm capability.

This can be also proven by means of a graphical representation. In Figs. 2 and 3 the $\alpha$-cuts of the joint PDs induced by the optimal Frank and GDO $t$-norms are plotted and compared with the alpha cuts of the reference joint PD. Figure 2 shows that the $\alpha$-cuts provided by the Frank $t$-norm are larger than the reference ones for low $\alpha$ values, while they

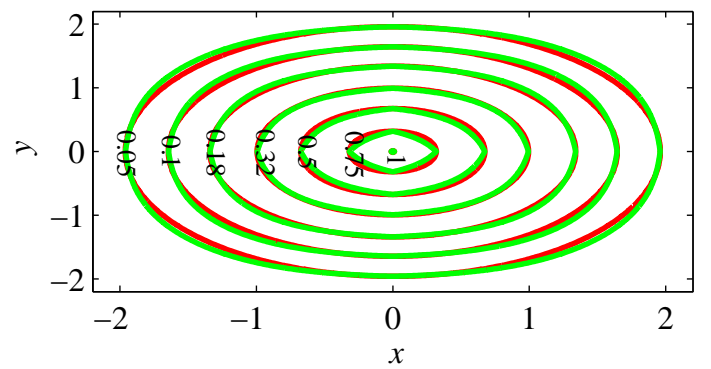

Figure 2: Comparison of the $\alpha$-cuts of the joint PD induced by $T_{\gamma=0.05}^{F}$ (green lines) with the $\alpha$-cuts of the reference joint PD (red lines). Standard Normal distributions are assumed for $p_{X}$ and $p_{Y}$.

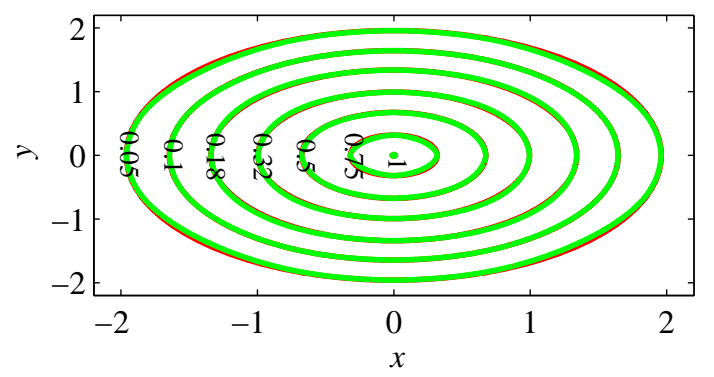

Figure 3: Comparison of the $\alpha$-cuts of the joint PD induced by $T_{\gamma_{1}=0.55, \gamma_{2}=1.47}^{\mathrm{GDO}}$ (green lines) with the $\alpha$ cuts of the reference joint PD (red lines). Standard Normal distributions are assumed for $p_{X}$ and $p_{Y}$.

are smaller than the reference ones for high $\alpha$ values. This means that the Frank $t$-norm introduces an evident systematic error in the evaluation of the joint PD. According to Fig. 3, also the GDO provides an approximated joint $\mathrm{PD}$, but the resulting $\alpha$-cuts are more similar to the reference $\alpha$-cuts.

\section{Example of uncertainty combination}

In the previous Section, an approximated method for the combination of random contributions to uncertainty based on $t$-norms has been discussed. In order to understand what is the effect of the approximation on the final uncertainty estimate, a very simple measurement example is here considered. Let measurand $z_{N}$ be the sum of $N$ measured samples $x_{i}$ :

$$
z_{N}=\sum_{i=1}^{N} x_{i}
$$

The measured values $x_{i}$ are assumed to be affected by a random noise such that a standard normal distribution $X_{i}$ can be associated to all of them to identify the possible measured values:

$$
X_{i}=\mathcal{N}(0,1)
$$

Both the standard approach to uncertainty defined in the GUM [1] and the RFV approach to 


\begin{tabular}{|c|c|c|}
\hline$N$ & $T_{\gamma=0.05}^{F}$ & $T_{\gamma_{1}=0.55, \gamma_{2}=1.47}^{\mathrm{GDO}}$ \\
\hline 2 & $4.1 \%$ & $1.0 \%$ \\
8 & $16.7 \%$ & $4.3 \%$ \\
32 & $35.2 \%$ & $10.4 \%$ \\
128 & $54.9 \%$ & $19.6 \%$ \\
\hline
\end{tabular}

Table 2: Errors $e$ introduced by $T_{\gamma}^{F}$ and $T_{\gamma_{1}, \gamma_{2}}^{\mathrm{GDO}}$ for different $N$ values.

uncertainty can be applied to the considered example to compute the resulting $Z_{N}$ distribution. Within the GUM approach, the $Z_{N}$ distribution is assumed to be a Normal PDF whose standard deviation $u_{C}\left(z_{N}\right)$ can be obtained by means of the Law of Propagation of Uncertainty (LPU) [1] that, for this example, yields:

$$
u_{C}\left(z_{N}\right)=\sqrt{\sum_{i=1}^{N} u^{2}\left(x_{i}\right)}=\sqrt{N} u(x)
$$

where $u(x)$ is the standard uncertainty of each $x_{i}$ that, in the considered example, is unitary. The resulting $\mathrm{PDF} Z_{N}$ is transformed into the $\mathrm{PD} \hat{Z}_{N}$ according to the 1-D transformation discussed in $[12,13]$. Since the GUM assumptions about the $Z_{N}$ normality are totally met for this particular example, $\hat{Z}_{N}$ can be considered as the reference PD.

Following the RFV approach, an RFV $\tilde{X}_{i}$ can be associated with each $x_{i}$. Actually, $\tilde{X}_{i}$ is an RFV composed by its random PD only, since only random contributions are considered in this example. Of course, the random PD is obtained by transforming the PDF $X_{i}$ according to the 1-D transformation. Once the random PDs $\tilde{X}_{i}$ are obtained, following (2) and (4) they can be composed to provide the random PD $\tilde{Z}_{N}$.

The reference $\mathrm{PD} \hat{Z}_{N}$ and the random $\mathrm{PD} \tilde{Z}_{N}$ are plotted in Fig. 4 for different numbers of samples $N$. In the left plots, $\tilde{Z}_{N}$ has been obtained by means of the Frank $t$-norm $T_{\gamma=0.05}^{F}$, while in the right plots $\tilde{Z}_{N}$ has been obtained by means of the GDO $T_{\gamma_{1}=0.55, \gamma_{2}=1.47}^{\mathrm{GDO}}$. The resulting normalized squared errors $e$ of the $\tilde{Z}_{N}$ estimates are reported in Table 2.

These results confirm that the GDO provides a more accurate uncertainty estimate than the Frank $t$-norm for all considered number of samples $N$. For $N>2$ the Frank $t$-norm provides an evident underestimate of uncertainty, while the estimate provided by the GDO is closer to the reference estimate. However, for $N=128$ also the GDO leads to an underestimate of uncertainty, especially for high $\alpha$ values (low confidence levels), and consequently, to a large error $(e=19.6 \%)$.

Better uncertainty estimates can be obtained for large $N$ values. In fact, the PDs shown in Fig. 4 have been obtained by applying the Frank $t$-norm and the GDO resulting from the optimization pro-

\begin{tabular}{|c|c|c|}
\hline$N$ & $T_{\gamma=0.01}^{F}$ & $T_{\gamma_{1}=1.1, \gamma_{2}=1.7}^{\mathrm{GDO}}$ \\
\hline 2 & $5.9 \%$ & $2.4 \%$ \\
8 & $15.9 \%$ & $3.3 \%$ \\
32 & $23.8 \%$ & $2.7 \%$ \\
128 & $35.2 \%$ & $4.4 \%$ \\
\hline
\end{tabular}

Table 3: Errors $e$ introduced by $T_{\gamma}^{F}$ and $T_{\gamma_{1}, \gamma_{2}}^{\mathrm{GDO}}$ for different $N$ values.

cedure discussed in Sec. 3.2, which is devoted to optimize the combination of two samples $x_{i}$ only. In other words, the optimal parameters obtained in Sec. 3.2 minimize only the error in the $\tilde{Z}_{2}$ estimate.

Different results can be obtained by applying the optimal parameters which minimize the error in a different $\tilde{Z}_{N}$ estimate. As an example, Fig. 5 shows the PDs resulting from the minimization of the error in $\tilde{Z_{32}}$. In the left plots of Fig. $5, \tilde{Z}_{N}$ has been obtained by means of the Frank $t$-norm $T_{\gamma=0.01}^{F}$, while in the right plots $\tilde{Z}_{N}$ has been obtained by means of the GDO $T_{\gamma_{1}=1.1, \gamma_{2}=1.7}^{\mathrm{GDO}}$. The resulting normalized squared error $e$ of the $\tilde{Z}_{N}$ estimates are reported in Table 3.

These results show that for the GDO it is possible to find some parameter values which drastically reduce the maximum error obtained for the considered numbers $N$. In fact, the maximum error is now limited to $e=4.4 \%$. On the contrary, this is not possible for the Frank $t$-norm, for which the error values remain too large. This can be explained considering that the GDO is a two-parameter $t$-norm family, while the Frank family is function of one parameter only. Therefore, the GDO can be more easily adapted to minimize the errors in the uncertainty estimates in the given range $2 \leq N \leq 128$.

However, even if optimized, the uncertainty estimate provided by the GDO is always an approximation of the correct uncertainty estimate. Therefore, also considering that the effectiveness of the approximation decreases as the number of samples $N$ increases, it is always possible to find a number $N$ for which the approximation is not acceptable.

\section{Conclusions}

This paper has proved that the combination of random contributions to uncertainty represented in terms of possibility distributions yields to approximate results and the choice of the $t$-norm employed to combine them is a critical step to ensure acceptable results.

While apparently good results can be obtained when only two results are combined, by means of relatively simple operators such as the Frank $t$-norm family, the residual approximation error may become soon unacceptable when more terms are combined.

The more complex generalized Dombi operator, 

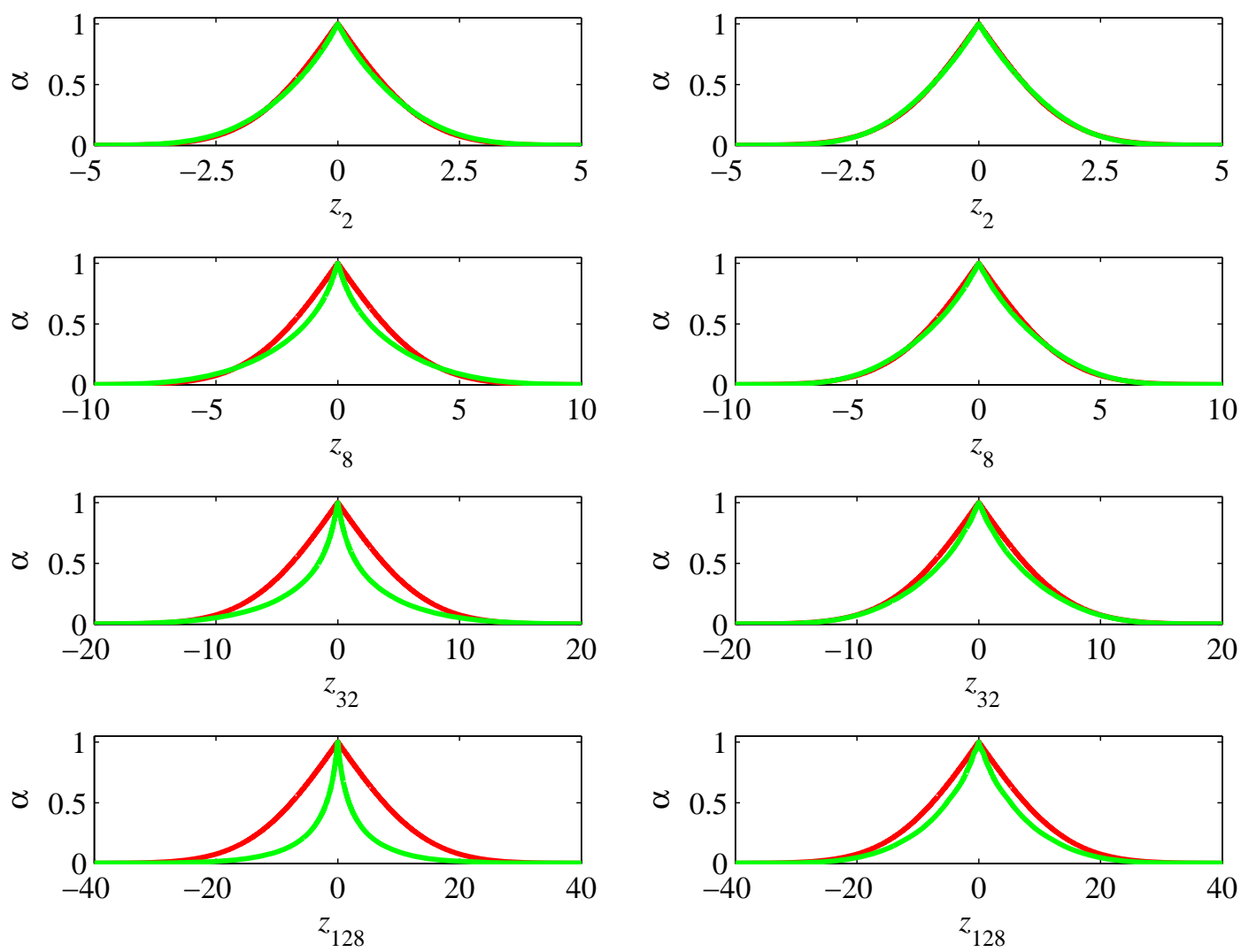

Figure 4: Reference PDs $\hat{Z}_{N}$ (red lines) and random PD $\tilde{Z}_{N}$ (green lines) for different $N$ values. In the left plots $T_{\gamma=0.05}^{F}$ has been applied, while in the right plots $T_{\gamma_{1}=0.55, \gamma_{2}=1.47}^{\mathrm{GDO}}$ has been applied.

belonging to a family of two-parameter $t$-norms, has been considered and it proved to return significantly lower approximation errors. However, the residual error may still originate large errors when several terms are combined together, as it may occur in complex measurement procedures.

The flexibility offered by two parameters, on the other hand, allows a more efficient optimization of the Dombi operator, so that acceptable solutions can be found as a function of the number of elements to be combined. It can be then concluded that the considered generalized Dombi operator represents an efficient solution to the combination of random contributions to uncertainty when they are represented in terms of possibility distributions.

\section{References}

[1] JCGM 100:2008. Evaluation of Measurement Data - Guide to the Expression of Uncertainty in Measurement, (GUM 1995 with minor corrections). Joint Committee for Guides in Metrology, 2008.

[2] A. Ferrero and S. Salicone. Uncertainty: Only one mathematical approach to its evaluation and expression? IEEE Trans. Instrum. Meas., 61(8):2167-2178, 2012.
[3] JCGM 200:2012. International Vocabulary of Metrology - Basic and General Concepts and Associated Terms (VIM 2008 with minor corrections). Joint Committee for Guides in Metrology, 2012.

[4] G. Mauris, V. Lasserre, and L. Foulloy. A fuzzy approach for the expression of uncertainty in measurement. Measurement, 29:165-177, 2001.

[5] M. Urbanski and J. Wasowsky. Fuzzy approach to the theory of measurement inexactness. Measurement, Elsevier Science, 34:67-74, 2003.

[6] A. Ferrero and S. Salicone. The random-fuzzy variables: a new approach for the expression of uncertainty in measurement. IEEE Trans. Instrum. Meas., 53(5):1370-1377, 2004.

[7] S. Salicone. Measurement Uncertainty: an approach via the mathematical theory of evidence. Springer series in reliability engineering. Springer, New York, NY, USA, 2007.

[8] A. Ferrero, M. Prioli, and S. Salicone. The construction of joint possibility distributions of random contributions to uncertainty. IEEE Trans. Instrum. Meas., 63(1):80-88, 2014.

[9] J. Dombi. Towards a general class of operators for fuzzy systems. IEEE Trans. Fuzzy Syst., 16(2):477-484, 2008. 

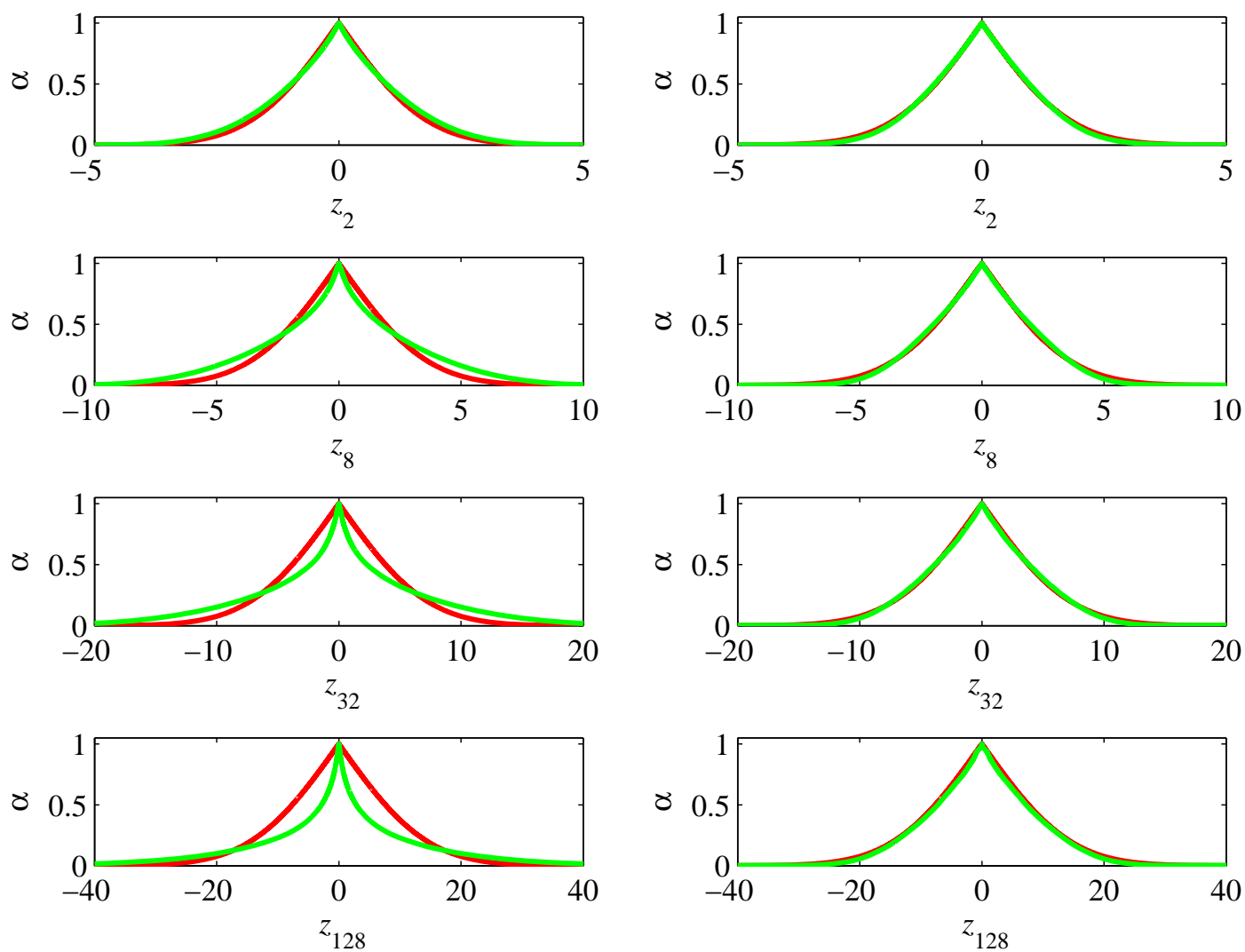

Figure 5: Reference PDs $\hat{Z}_{N}$ (red lines) and random PD $\tilde{Z}_{N}$ (green lines) for different $N$ values. In the left plots $T_{\gamma=0.01}^{F}$ has been applied, while in the right plots $T_{\gamma_{1}=1.1, \gamma_{2}=1.7}^{\mathrm{GDO}}$ has been applied.

[10] A. Ferrero and S. Salicone. The construction of random-fuzzy variables from the available relevant metrological information. IEEE Trans. Instrum. Meas., 58(2):365-374, 2009.

[11] A. Ferrero, M. Prioli, and S. Salicone. Processing dependent systematic contributions to measurement uncertainty. IEEE Trans. Instrum. Meas., 62(4):720-731, 2013.

[12] D. Dubois, L. Foulloy, G. Mauris, and H. Prade. Probability-possibility transformations, triangular fuzzy sets, and probabilistic inequalities. Reliable Computing. Kluwer Academic Publishers, 10:273-297, 2004.

[13] A. Ferrero, M. Prioli, S. Salicone, and B. Vantaggi. 2D probability-possibility transformations. In Synergies of Soft Computing and Statistics for Intelligent Data Analysis, volume 190 of Advances in Intelligent Systems and Computing, pages 63-72. Springer Berlin Heidelberg, 2013.

[14] A.Ferrero, M. Prioli, and S. Salicone. Uncertainty propagation through non-linear measurement functions by means of joint randomfuzzy variables. In I2MTC 2015, Pisa, Italy, May 11-14, 2015.

[15] Erich Peter Klement, Radko Mesiar, and Endre
Pap. Triangular norms. position paper I: basic analytical and algebraic properties. Fuzzy Sets and Systems, 143(1):5 - 26, 2004.

[16] L. A. Zadeh. Fuzzy sets as a basis for a theory of possibility. Fuzzy Sets and Systems, 1(1):328, 1978 .

[17] A. Ferrero, M. Prioli, and S. Salicone. Conditional random-fuzzy variables representing measurement results. IEEE Trans. Instrum. Meas., 64(5):1170-1178, 2015.

[18] D. Dubois and H. Prade. Bayesian conditioning in possibility theory. Fuzzy Sets and Systems, 92(2):223-240, 1997.

[19] C. Alsina, M. J. Frank, and B. Schweizer. Associative Functions: Triangular Norms and Copulas. World Scientific, 2006.

[20] Erich Peter Klement, Radko Mesiar, and Endre Pap. Triangular norms. position paper II: general constructions and parameterized families. Fuzzy Sets and Systems, 145(3):411 - 438, 2004.

[21] A. Ferrero, M. Prioli, and S. Salicone. The evaluation of uncertainty contributions due to uncompensated systematic effects. In I2MTC 2013, pages 1028-1033, Minneapolis, USA, May 6-9, 2013. 